EPJ Web of Conferences 75, 07005 (2014)

DOI: $10.1051 /$ epjconf/20147507005

(c) Owned by the authors, published by EDP Sciences, 2014

\title{
Vortex core properties in iron pnictides
}

\author{
I. Zakharchuk ${ }^{1,2, a}$, A. Sharafeev ${ }^{1,2}$, P. Belova ${ }^{1,3}$, K. B. Traito ${ }^{1}$, and E. Lähderanta ${ }^{1}$ \\ ${ }^{1}$ Lappeenranta University of Technology, P.O. Box 20, FI-53851, Lappeenranta, Finland \\ ${ }^{2}$ Saint-Petersburg Electrotechnical University, Popov str. 5, RU-197376, St.Petersburg, Russia \\ ${ }^{3}$ Petrozavodsk State University, Lenin str. 33, RU-185640, Petrozavodsk, Russia
}

\begin{abstract}
The mechanism of unconventional superconductivity in recently discovered Fe-based superconductors has been intensively discussed. A plausible candidate is the superconducting (SC) pairing mediated by antiferromagnetic (AFM) interactions. There are two different approaches predicting the $s^{ \pm}$pairing state, in which the SC gap shows an $s$-wave symmetry that changes sign between different Fermi-surface (FS) sheets. The first one is based on the itinerant spin fluctuations promoted by FS nesting, and the second is based on the local AFM exchange couplings. We apply quasiclassical Eilenberger approach to the vortex state to calculate the cutoff parameter, $\xi_{h}$, at different levels of impurity scattering rates and to compare results with experimental data for iron pnictides. The $s^{ \pm}$-wave pairing symmetry is considered as a presumable state for these materials. Magnetic field dependence of $\xi_{h} / \xi_{c 2}$ is found to be nonuniversal for $s^{ \pm}$pairing: depending on the chosen parameter set it can reside both below and above analytical Ginzburg-Landau curve. It is also found that normalized $\xi_{2} / \xi_{c 2}\left(B / B_{c 2}\right)$ dependence is increasing with pair-breaking (interband) impurity scattering, and the intraband scattering results in decreasing of the $\xi_{2} / \xi_{c 2}$ value. Here, $\xi_{2}$ is the vortex core size and $\xi_{c 2}$ is the GinzburgLandau coherence length determined from the upper critical field. The $\xi_{2} / \xi_{c 2}\left(B / B_{c 2}\right)$ curve has a minimum at low temperatures and small scattering evolving into monotonously decreasing function at strong scattering and high temperatures.
\end{abstract}

\section{Introduction}

In iron pnictides, superconductivity and magnetism are close neighbors on the phase diagram, and it has been proposed $[1,2]$ that the pairing mechanism is due to AFM spin-fluctuation exchange, similar to high- $T_{c}$ cuprate superconductors. However, the geometry of low-energy states in iron-based pnictides and in the cuprates is different, and in most ferropnicitdes the momentum $\mathbf{Q}$ connects low-energy fermionic states near the center and the corner of the Brillouin zone. Thus the SC gap $\Delta(\mathbf{k})$ must be symmetric with respect to $k_{x} \rightarrow k_{y}$ and $k_{x} \rightarrow-k_{x}$, but still change the sign under $\mathbf{k} \rightarrow \mathbf{k}+\mathbf{Q}$. Such gap is generally called an extended $s$-wave gap, or $s^{ \pm}$gap. Strong-coupling approaches to pairing, based on the AFM local $J_{1}-J_{2}$ multiple competing exchange model of the magnetism in these systems, are also often used [3]. In that study, the exchange terms have been decoupled in the mean field in the pairing channel. In the region of the general phase diagram with $J_{2} \gtrsim J_{1}, \cos k_{x} \cos k_{y}$ was the leading instability for the 2-band Fermi surface, leading to a nodeless $s^{ \pm}$state. Experimental evidence for $s^{ \pm}$pairing symmetry has been circumstantially reviewed in Ref. [4].

In spite of success of $s^{ \pm}$model, there are some indications that a conventional $s$-wave state without sign reversal $\left(s_{++}\right.$-wave state) is also a possible candidate for iron

\footnotetext{
ae-mail: ivan.zakharchuk@lut.fi
}

pnictides. It has been proposed that the moderate electronphonon interaction due to $\mathrm{Fe}$-ion oscillation can induce the critical orbital fluctuation, without being prohibited by the Coulomb interaction [5].

Important information about the order parameter symmetry can be obtained from the investigation of the mixed state. The scanning tunneling microscopy (STM) experiments probe the spatial variation of the local density of states [6], whereas $\mu \mathrm{SR}$ is sensitive to the spatial dependence of the local internal magnetic field $B(r)$ [7]. The vortex core size is determined from the $\mu \mathrm{SR}$ measurements by fitting them to a theoretical function for $B(r)$ that includes a cutoff function $F\left(\mathbf{G}, \xi_{h}\right)$, where $\mathbf{G}$ are the reciprocal lattice vectors and $\xi_{h}$ is the cutoff parameter. The functional form of $F\left(\mathbf{G}, \xi_{h}\right)$ depends on the spatial dependence of the superconducting order parameter $\Delta(r)$ in the core region. Consequently, cutoff parameter $\xi_{h}$ is generally not the coherence length, but rather a measure of the vortex core size. A definition of the vortex core size is the radius $\xi_{2}$ at which the supercurrent density $|J(r)|$ calculated from $B(r)$ reaches its maximum.

\section{Model}

Following the microscopical Eilenberger theory, the cutoff parameter, $\xi_{h}$, can be found from the fitting of the calculated magnetic field distribution $h_{E}(\mathbf{r})$ to the Eilenberger - 
Hao-Clem (EHC) field distribution $h_{E H C}(\mathbf{r})$ [8]

$$
h_{E H C}(\mathbf{r})=\frac{\Phi_{0}}{S} \sum_{\mathbf{G}} \frac{F(G) e^{i \mathbf{G r}}}{1+\lambda^{2} G^{2}} .
$$

Here,

$$
F(\mathbf{G})=u K_{1}(u),
$$

where $K_{1}(u)$ is modified Bessel function, $u=\xi_{h} G, S$ is the area of the vortex lattice unit cell and $\lambda(T)$ is the penetration depth in the Meissner state.

The magnetic-field penetration depth $\lambda(T)$ is assumed to be field independent and to have the same value as in the Meissner state. In this approach all field dependent effects are taken into account in $\xi_{h}(B)$ dependence. The London penetration depth can be experimentally measured with great precision and its variation with temperature depends sensitively on the gap structure. For $T=T_{c} / 3$, a conventional isotropic $s$-wave gap $\Delta_{0}$ results in an exponential behavior, $\Delta \lambda(T) \propto \exp \left(-\Delta_{0} / T\right)$, which is preserved even with the addition of non-magnetic impurities. Unconventional pairing states, on the other hand, are susceptible to the presence of non-magnetic impurities, which result in power-law behavior [9].

The penetration depth for $s^{ \pm}$-wave superconductor was calculated in Born approximation for impurity scattering in Ref. [9]

$$
\frac{\lambda_{L 0}^{2}}{\lambda^{2}(T)}=2 \pi T \sum_{\omega_{n}>0} \frac{\bar{\Delta}_{n}^{2}}{\eta_{n}\left(\bar{\Delta}_{n}^{2}+\omega_{n}^{2}\right)^{3 / 2}},
$$

where $\lambda_{L 0}$ is the London penetration depth at $T=0$ $\mathrm{K}$ in the absence of the impurities and $\eta_{n}=1+2 \pi *$ $\left(\Gamma_{0}+\Gamma_{\pi}\right) /\left(\sqrt{\bar{\Delta}_{n}^{2}+\omega_{n}^{2}}\right)$. Here, $\Gamma_{0}=\pi n_{i} N_{F}\left|u_{0}\right|^{2}$ and $\Gamma_{\pi}=$ $\pi n_{i} N_{F}\left|u_{\pi}\right|^{2}$ are the intra- and interband impurity scattering rates, respectively $\left(u_{0, \pi}\right.$ are impurity scattering amplitudes with correspondingly small, or close to $\pi=(\pi, \pi)$, momentum transfer). In Eq. (3), $\bar{\Delta}_{n}=\Delta(T)-4 \pi \Gamma_{\pi} \bar{\Delta}_{n} / \sqrt{\bar{\Delta}_{n}^{2}+\omega_{n}^{2}}$ for the $s^{ \pm}$pairing and $\bar{\Delta}_{n}=\Delta(T)$ for the $s_{++}$pairing symmetry. The order parameter $\Delta(T)$ in Meissner state is determined by the self-consistent equation

$$
\Delta(T)=2 \pi T \sum_{0<\omega_{n}<\omega_{c}} \frac{V^{S C} \bar{\Delta}_{n}}{\sqrt{\bar{\Delta}_{n}^{2}+\omega_{n}^{2}}} .
$$

As was shown in Ref. $[9,10]$ this model of scattering reasonably explains experimental results.

The Eilenberger equations for quasiclassical functions $f$ and $g$ under Riccati parametrization can be written as [8]

$$
\begin{aligned}
\mathbf{u} \cdot \nabla a & =-a\left[2\left(\omega_{n}+G\right)+i \mathbf{u} \cdot \mathbf{A}_{E}\right] \\
& +(\Delta+F)-a^{2}\left(\Delta^{*}+F^{*}\right), \\
\mathbf{u} \cdot \nabla b & =b\left[2\left(\omega_{n}+G\right)+i \mathbf{u} \cdot \mathbf{A}_{E}\right] \\
& -\left(\Delta^{*}+F^{*}\right)+b^{2}(\Delta+F),
\end{aligned}
$$

where $\omega_{n}=\pi T(2 n+1), G=2 \pi\langle g\rangle\left(\Gamma_{0}+\Gamma_{\pi}\right) \equiv 2 \pi\langle g\rangle \Gamma^{*}$, $F=2 \pi\langle f\rangle\left(\Gamma_{0}-\Gamma_{\pi}\right)$ for $s^{ \pm}$pairing symmetry and $F=2 \pi\langle f\rangle \Gamma^{*}$ for the $s_{++}$pairing symmetry. Here, Born approximation for impurity scattering is used and the pairing potential $\Delta(\mathbf{r})$ in the vortex state is determined selfconsistently by function $f$.
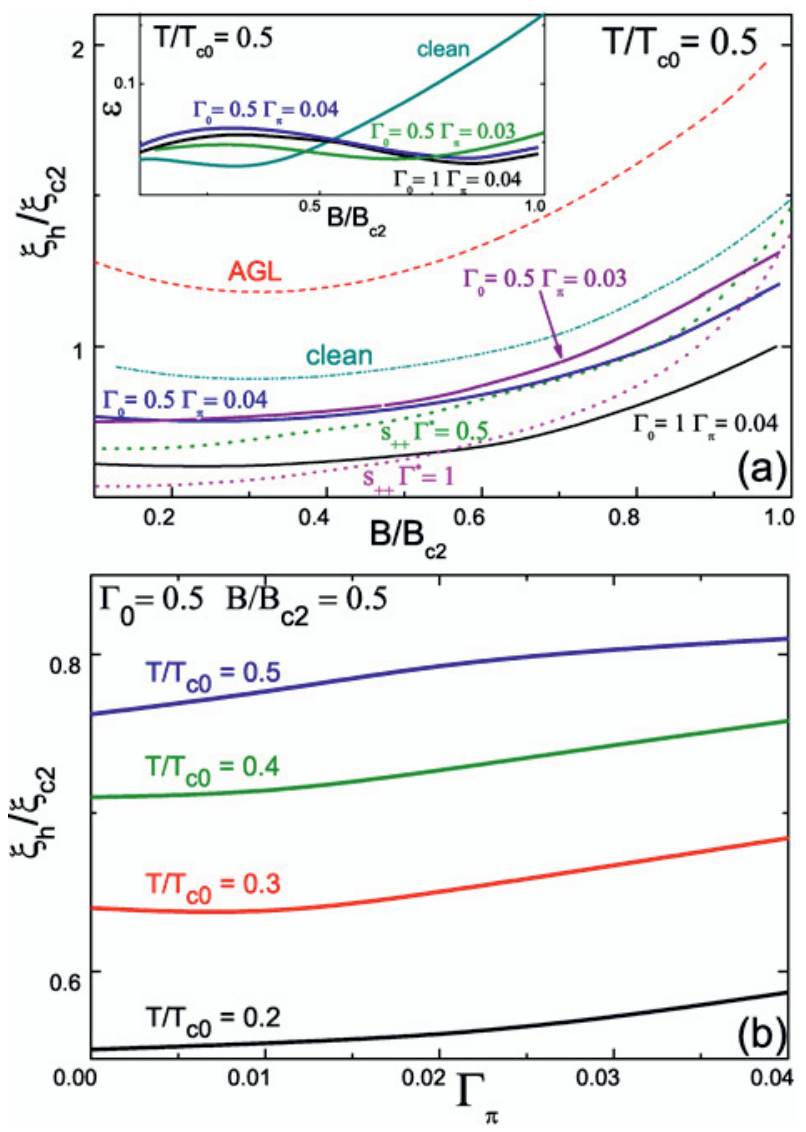

Figure 1. (Color online) (a) The magnetic field dependence of $\xi_{h} / \xi_{c 2}$ for superconductors with impurity scattering. The dashdotted and solid lines represent our solution of Eilenberger equations at $T / T_{c 0}=0.5$ for "clean" case $\left(\Gamma_{0}=\Gamma_{\pi}=0\right)$ and $s^{ \pm}$ model $\left(\Gamma_{0}=0.5, \Gamma_{\pi}=0.03 ; \Gamma_{0}=0.5, \Gamma_{\pi}=0.04\right.$ and $\Gamma_{0}=1$, $\left.\Gamma_{\pi}=0.04\right)$, correspondingly. The dotted lines show result for $s_{++}$model $\left(\Gamma^{*}=0.5\right.$ and $\left.\Gamma^{*}=1\right)$. Dashed line demonstrates the result of the AGL theory for $\xi_{v}$ from Eq. 7. The inset shows the magnetic field dependence of mean square deviation of the $h_{E H C}$ distribution from the Eilenberger distribution normalized by the variance of the Eilenberger distribution, $\varepsilon$, at $T / T_{c 0}=0.5$ for $\Gamma_{0}=\Gamma_{\pi}=0$ ("clean"); $\Gamma_{0}=0.5, \Gamma_{\pi}=0.03 ; \Gamma_{0}=0.5$, $\Gamma_{\pi}=0.04$ and $\Gamma_{0}=1, \Gamma_{\pi}=0.04$. (b) The interband scattering $\Gamma_{\pi}$ dependence of $\xi_{h} / \xi_{c 2}$ at different temperatures $T / T_{c 0}$ (intraband scattering $\Gamma_{0}=0.5$ and $B / B_{c 2}=0.5$ ) for the $s^{ \pm}$pairing.

\section{Results}

Using the similarity to the model of spin-flip superconductors, $B_{c 2}(T)$ for two-dimensional $s^{ \pm}$pairing was determined in Ref. [11]. Dashed line in Fig. 1 (a) demonstrates the result of analytical Ginzburg-Landau (AGL) model for $\xi_{v}[7]$

$$
\xi_{v}=\xi_{c 2}\left(\sqrt{2}-\frac{0.75}{\kappa_{G L}}\right)\left(1+b^{4}\right)^{1 / 2}\left[1-2 b(1-b)^{2}\right]^{1 / 2} .
$$

Here, we use the notation of the AGL theory for the cutoff parameter, $\xi_{v}$ instead of $\xi_{h}$, and $b=B / B_{c 2}$. This dependence with $\xi_{c 2}$ as a fitting parameter is often used for the description of the experimental $\mu \mathrm{SR}$ results [7]. As can be seen from Fig. 1 (a), the magnetic field dependence of $\xi_{h} / \xi_{c 2}$ is nonuniversal because it depends not 
only on $B / B_{c 2}$ (as in the AGL theory, dashed line in Fig. 1 (a)), but also on interband and intraband impurity scattering parameters. In the clean case where $\Gamma_{0}=\Gamma_{\pi}=0$, the results are similar for $s^{ \pm}$and $s_{++}$pairing symmetries. We indicated that this curve is "clean" one. In this figure, the case $\Gamma_{0} \gg \Gamma_{\pi}$ is considered and the value of $\xi_{h}$ is reduced considerably in comparison with the clean case. One can compare the observed behavior with that in $s_{++}$pairing model. In $s_{++}$pairing symmetry the intraband and interband scattering rates act in a similar way and $\xi_{h} / \xi_{c 2}$ decreases always with impurity scattering. In contrast, in $s^{ \pm}$model $\xi_{h} / \xi_{c 2}\left(B / B_{c 2}\right)$ curves show different forms of behavior with $\Gamma_{\pi}$. A crossing point appears in the $\xi_{h} / \xi_{c 2}\left(B / B_{c 2}\right)$ dependences for $s^{ \pm}$and $s_{++}$pairings. In case of $\Gamma^{*}=\Gamma_{0}=0.5$ and $\Gamma_{\pi}=0.04, \xi_{h} / \xi_{c 2}$ increases regarding $s_{++}$values at $B / B_{c 2}<0.8$ and decreases at higher fields becoming more flattened. A similar effect is visible for $\Gamma^{*}=\Gamma_{0}=1$ with the crossing point at $B / B_{c 2} \sim 0.55$. We also calculated the magnetic field dependence of mean square deviation of $h_{E H C}$ distribution of the magnetic field from the Eilenberger distribution normalized by the variance of the Eilenberger distribution $\varepsilon=\sqrt{\overline{\left(h_{E}-h_{E H C}\right)^{2}} / \overline{\left(h_{E}-B\right)^{2}}}$, where $\bar{\cdots}$ is the average over a unit vortex cell. It can be seen in the inset to Fig. 1 (a) that the accuracy of effective London model is deteriorating as the magnetic field increases; however, in superconductors with impurity scattering the error is below $6 \%$ even when it is close to the second critical field.

In Fig. 1 (b), the interband scattering $\Gamma_{\pi}$ dependences of $\xi_{h}$ are presented in low fields for the $s^{ \pm}$pairing at different temperatures $T / T_{c 0}$. As can be seen $\xi_{h} / \xi_{c 2}$ increases with the interband scattering rate $\Gamma_{\pi}$. Strong decreasing of $\xi_{h} / \xi_{c 2}$ with a decrease in the temperature can be explained by the Kramer-Pesch effect. Recently, the image of the vortices in a wide field range from $0.1 \mathrm{~T}$ to $11 \mathrm{~T}$ by mapping the tunneling conductance at the Fermi energy in LiFeAs compound was obtained [12]. It was found that the vortex radius shrank with decreasing temperature and became smaller than the coherence length estimated from the upper critical field. This effect was considered as a direct evidence of the Kramer-Pesch effect expected in a clean superconductor [12]. It should be noted that the normalization constant $\xi_{c 2}$ increases with $\Gamma_{\pi}$ because $\Gamma_{\pi}$ suppress $T_{c}$ similar to superconductors with spin-flip scattering (violation of the Anderson theorem). Thus, rising of $\xi_{h} / \xi_{c 2}$ implies more strong growth of $\xi_{h}$ than $\xi_{c 2}$ (from GL theory one would expect $\xi_{h} / \xi_{c 2}=$ Const). Qualitatively, it can be explained by the strong temperature dependence of $\xi_{h}\left(B, T / T_{c}\right)$, which is connected to the Kramer-Pesch effect. Increasing $\Gamma_{\pi}$ results in suppression of $T_{c}$, i.e. effective increasing of $T$ and $\xi_{h}\left(T / T_{c}\right)$. The $\xi_{c 2}\left(T / T_{c}\right)$ does not have such strong $T_{c}$ dependence, thus leading to the increase of $\xi_{h} / \xi_{c 2}$ ratio with $\Gamma_{\pi}$. A small value of the cutoff parameter $\left(\xi_{h} / \xi_{c 2} \sim 0.4\right.$ at $\left.T / T_{c}=0.18\right)$ was observed in iron pnictide $\mathrm{BaFe}_{1.82} \mathrm{Co}_{0.18} \mathrm{As}$ [13], which is comparable with our theoretical prediction, Fig. 1 (b). In Ref. [13] the magnetic field distribution shape of the sample was explained by effects of field-induced magnetic order and vortex-lattice disorder. Our consideration shows the im- portance of impurity scattering even in the triangular lattice giving second possible explanation for the experimental results.

We also study the case of weak intraband scattering. This case can be realized in stoichiometrical pnictides such as LiFeAs. A high value $\xi_{h}(B \rightarrow 0, T=0)=9.8 \mathrm{~nm}$ was obtained in SANS measurements in LiFeAs [14] in comparison with $\xi_{c 2}(T=0) \approx 4.6 \mathrm{~nm}$ [15], i.e. $\xi_{h} / \xi_{c 2} \approx 2.1$. For the simplicity we considered the same scattering parameters $\Gamma_{0}=\Gamma_{\pi}=\Gamma$. As can be seen from the inset to Fig. 2, the shape of $\xi_{h} / \xi_{c 2}\left(B / B_{c 2}\right)$ curve does not change considerably, but the absolute values of $\xi_{h} / \xi_{c 2}$ depend crucially on $\Gamma$. At low $\Gamma$ values, $\xi_{h} / \xi_{c 2}$ resides below the AGL curve and moves above it at high $\Gamma$ (for gapless superconductivity case $\Gamma>0.064$ ). Such behavior is quite different from that in $s_{++}$pairing symmetry where intraband and interband scattering rates act in similar way and $\xi_{h} / \xi_{c 2}$ decreases always with impurity scattering. The obtained $\xi_{h}(B \rightarrow 0) / \xi_{c 2}=1.8$ in the $s^{ \pm}$model is much more near to the experimental results[14] than in the $s_{++}$ model, where strong reduction of $\xi_{h}$ is visible. Taking into account two different nodeless gaps found by scanning tunneling spectroscopy [16] can also improve the theory and experiments matching. The value of half-width-athalf-maximum (HWHM) of tunneling-conductance vortex images of $\mathrm{LiFeAs} \sim 2.5 \mathrm{~nm}$ at $T \rightarrow 0$ [12], i.e. $\mathrm{HWHM} / \xi_{c 2} \approx 0.54$ comparable to our results $\xi_{2} / \xi_{c 2}$. The

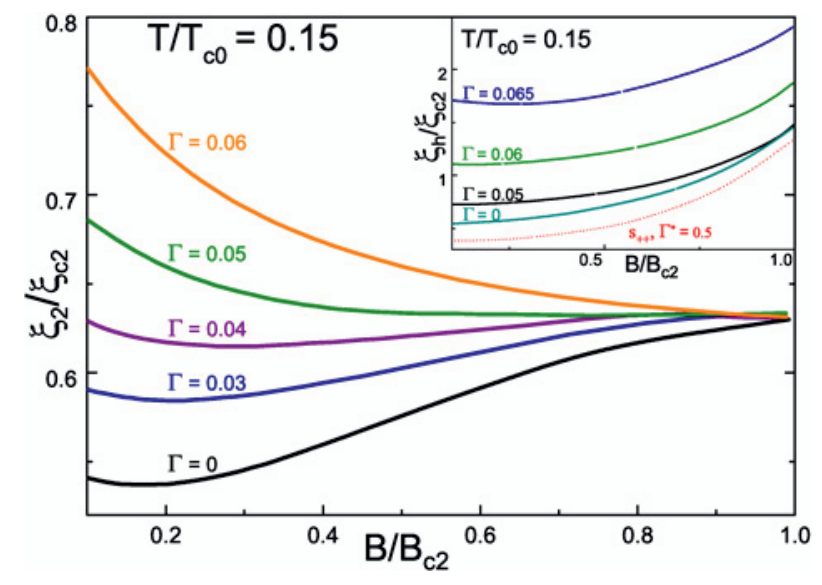

Figure 2. (Color online) The magnetic field dependence of $\xi_{2} / \xi_{c 2}$ with the same values of intraband $\Gamma_{0}$ and interband $\Gamma_{\pi}$ scattering rate $\Gamma(\Gamma=0,0.03,0.04,0.05,0.06)$ at $T / T_{c 0}=0.15$ for $s^{ \pm}$pairing symmetry. The inset shows magnetic field dependence of the cutoff parameter at $T / T_{c 0}=0.15$ with the same values of intraband $\Gamma_{0}$ and interband $\Gamma_{\pi}$ scattering rates $\Gamma(\Gamma=0$ for "clean" case and $\Gamma=0.05,0.06,0.065$ for the $s^{ \pm}$pairing). Dotted line shows result for $s_{++}$model $\left(\Gamma^{*}=0.5\right)$.

difference between pairing symmetries reveals itself in impurity scattering dependence of $\xi_{h} / \xi_{c 2}$. In $s_{++}$symmetry $\xi_{h} / \xi_{c 2}$ always decreases with impurity scattering rate $\Gamma$ (Fig. 1 (a)), in $s^{ \pm}$symmetry its behavior depends on the field range and relative values of intraband and interband impurity scattering rates: it can be a decreasing function of $\Gamma_{\pi}$ [8] or an increasing function of $\Gamma_{\pi}$ (Fig. 1 (b)). Fig. 2 shows the magnetic field dependence of $\xi_{2} / \xi_{c 2}$ with dif- 


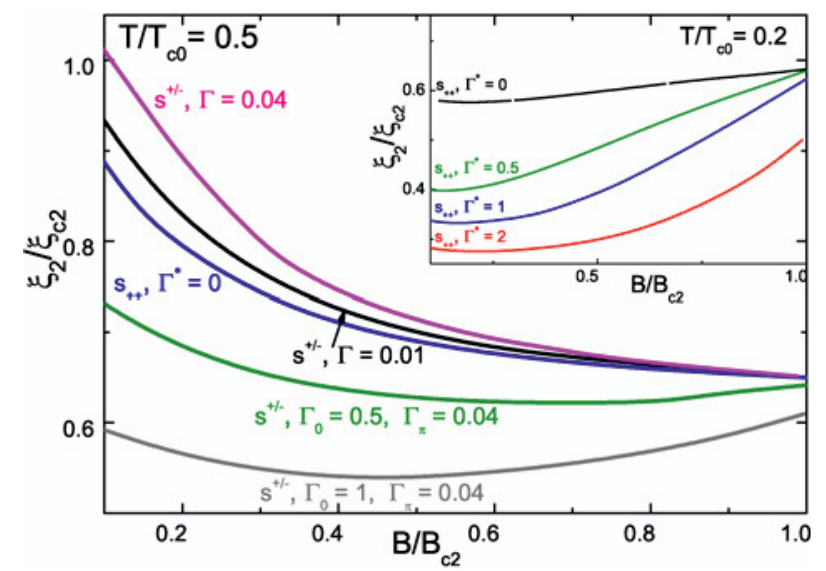

Figure 3. (Color online) The magnetic field dependence of $\xi_{2} / \xi_{c 2}$ with different impurity scattering rates $\Gamma$ with $T / T_{c 0}=0.5$ for $s_{++}$model $\left(\Gamma^{*}=0\right)$, for $s^{ \pm}$pairing symmetry $\left(\Gamma_{0}=\Gamma_{\pi}=\Gamma=\right.$ $0.01,0.04, \Gamma_{0}=0.5, \Gamma_{\pi}=0.04$ and $\left.\Gamma_{0}=1, \Gamma_{\pi}=0.04\right)$. The inset shows magnetic field dependence of $\xi_{2} / \xi_{c 2}$ at $T / T_{c 0}=0.2$ with different impurity scattering rates $(\Gamma=0,0.5,1,2)$ for $s_{++}$ symmetry.

ferent impurity scattering rates $\Gamma$ with $T / T_{c 0}=0.15$ for $s^{ \pm}$pairing symmetry. It can be seen from this figure that the normalized vortex core size increases monotonously with growth of impurity scattering rate. The shape of $\xi_{2} / \xi_{c 2}\left(B / B_{c 2}\right)$ curve also changes: curves with minimum at small $\Gamma$ transform to the monotonously decreasing field dependent function at high $\Gamma$, i.e. crossover from $d \xi_{2} / d B>$ 0 to $d \xi_{2} / d B<0$ behavior at $B=B_{c 2}$ occurs.

Fig. 3 depicts the magnetic field dependence of $\xi_{2} / \xi_{c 2}$ with different impurity scattering rates $\Gamma$ at $T / T_{c 0}=0.5$ for $s_{++}$model $\left(\Gamma^{*}=0\right)$, for $s^{ \pm}$pairing symmetry $\left(\Gamma_{0}=\right.$ $\Gamma_{\pi}=\Gamma=0.01,0.04, \Gamma_{0}=0.5, \Gamma_{\pi}=0.04$ and $\Gamma_{0}=1$, $\left.\Gamma_{\pi}=0.04\right)$. It can be seen from Fig. 3 that all considered pairing symmetries result in similar monotonously decreasing $\xi_{2} / \xi_{c 2}\left(B / B_{c 2}\right)$ dependence at weak impurity scattering rates and intermediate temperatures. The inset shows magnetic field dependence of $\xi_{2} / \xi_{c 2}$ at $T / T_{c 0}=0.2$ with different impurity scattering rates $(\Gamma=0,0.5,1,2)$ for $s_{++}$symmetry. Here, $\xi_{2} / \xi_{c 2}$ decreases with impurity scattering for $s_{++}$symmetry. To differentiate $s_{++}$and $s^{ \pm}$ symmetries the impurities creating interband scattering are needed (inset to Fig. 2).

\section{Conclusions}

In conclusion, Eilenberger equations have been solved for superconductors with $s^{ \pm}$and $s_{++}$pairing symmetries in the mixed state. It is found that normalized values of $\xi_{h} / \xi_{c 2}$ and $\xi_{2} / \xi_{c 2}$ decrease with temperature due to Kramer-Pesch effect. In unconventional superconductors, $\xi_{2} / \xi_{c 2}$ increases with pair-breaking impurity scattering.
The intraband scattering results in decreasing of $\xi_{2} / \xi_{c 2}$ value. The transformation from diminishing to growing of $\xi_{2} / \xi_{c 2}\left(B / B_{c 2}\right)$ dependence with lowering of the temperature is obtained for $s_{++}$-wave symmetry. A reasonable agreement between calculated $\xi_{h} / \xi_{c 2}$ and $\xi_{2} / \xi_{c 2}$ and those obtained experimentally in $\mathrm{Ba}\left(\mathrm{Fe}_{1-x} \mathrm{Co}_{x}\right)_{2} \mathrm{As}_{2}$ and $\mathrm{LiFeAs}$ is found.

This work was supported by the Finnish Cultural Foundation (Etelä-Karjalan rahasto).

\section{References}

1. I.I. Mazin, D.J. Singh, M.D. Johannes, M.H. Du, Phys. Rev. Lett. 101, 057003 (2008)

2. K. Kuroki, S. Onari, R. Arita, H. Usui, Y. Tanaka, H. Kontani, H. Aoki, Phys. Rev. Lett. 101, 087004 (2008)

3. K. Seo, B.A. Bernevig, J. Hu, Phys. Rev. Lett. 101, 206404 (2008)

4. P.J. Hirschfeld, M.M. Korshunov, I.I. Mazin, Rep. Prog. Phys. 74, 124508 (2011)

5. H. Kontani, S. Onari, Phys. Rev. Lett. 104, 157001 (2010)

6. Ø. Fischer, M. Kugler, I. Maggio-Aprile, C. Berthod, C. Renner, Rev. Mod. Phys. 79, 353 (2007)

7. J.E. Sonier, Rep. Prog. Phys. 70, 1717 (2007)

8. P. Belova, M. Safonchik, K.B. Traito, E. Lähderanta, Phys. Rev. B 83, 104518 (2011)

9. A.B. Vorontsov, M.G. Vavilov, A.V. Chubukov, Phys. Rev. B 79, 140507(R) (2009)

10. R. Prozorov, V.G. Kogan, Rep. Prog. Phys. 74, 124505 (2011)

11. Y.N. Ovchinnikov, V.Z. Kresin, Phys. Rev. B 52, 3075 (1995)

12. T. Hanaguri, K. Kitagawa, K. Matsubayashi, Y. Mazaki, Y. Uwatoko, H. Takagi, Phys. Rev. B 85, 214505 (2012)

13. J.E. Sonier, W. Huang, C.V. Kaiser, C. Cochrane, V. Pacradouni, S.A. Sabok-Sayr, M.D. Lumsden, B.C. Sales, M.A. McGuire, A.S. Sefat et al., Phys. Rev. Lett. 106, 127002 (2011)

14. D.S. Inosov, J.S. White, D.V. Evtushinsky, I.V. Morozov, A. Cameron, U. Stockert, V.B. Zabolotnyy, T.K. Kim, A.A. Kordyuk, S.V. Borisenko et al., Phys. Rev. Lett. 104, 187001 (2010)

15. N. Kurita, K. Kitagawa, K. Matsubayashi, A. Kismarahardja, E. Choi, J.S. Brooks, Y. Uwatoko, S. Uji, T. Terashima, J. Phys. Soc. Jpn. 80, 013706 (2011)

16. S. Chi, S. Grothe, R. Liang, P. Dosanjh, W.N. Hardy, S.A. Burke, D.A. Bonn, Y. Pennec, Phys. Rev. Lett. 109, 087002 (2012) 